\title{
EFFECTS OF MISALIGNMENTS ON SPACE-CHARGE-DOMINATED HEAVY ION BEAMS IN AN IRE
}

\author{
C.M. Celata and M.J.L. de Hoon, LBNL, Berkeley, CA,USA \\ J.J. Barnard, LLNL, Livermore, CA, USA
}

\begin{abstract}
Preliminary designs for the next large accelerator experiment envisioned for the Heavy Ion Fusion program, the IRE (Integrated Research Experiment) use an induction linac to accelerate multiple space-charge-dominated ion beams to an energy of several hundred $\mathrm{MeV}$, and focus them at a target. This paper examines the effect of beam and quadrupole misalignments on beam emittance in the IRE. The dependence of the centroid orbit on the scaling of focusing parameters with $\mathrm{z}$ is analyzed. PIC simulations including misalignment give acceptable emittance growth for present IRE designs.
\end{abstract}

\section{INTRODUCTION}

The Heavy Ion Fusion program is in the process of designing the Integrated Research Experiment (IRE), a multi-beam induction linac which would accelerate spacecharge-dominated driver-scale $\mathrm{K}^{+}$beams to a few hundred $\mathrm{MeV}$ and focus them onto a target. Preliminary linac designs consist of acceleration to $\sim 8 \mathrm{MeV}$ using electrostatic quadrupole focusing (IRE Section 1), followed by 2 sections of magnetic quadrupole focusing (IRE Sections 2 and 3). The phase advance per lattice period without space charge, $\sigma_{0}$, and beam radius are kept constant throughout the machine in order to maximize transportable current and minimize cost. This requires different scaling of focusing parameters in different sections. In Section $1, \mathrm{~L} / \mathrm{v}, \eta$, and $\mathrm{K}=\mathrm{qE} / \mathrm{m}$ are constant ( $\mathrm{L}=$ lattice half period length, $\mathrm{v}=$ longitudinal (i.e., z) velocity, $\eta \mathrm{L}=$ quadrupole length, and $\mathrm{E}^{\prime}=$ quadrupole gradient). In IRE Section 2, the beams are compressed longitudinally with line charge density, $\lambda, \propto v$. Here $\mathrm{L} \propto \mathrm{v}^{1 / 2}$, and $\eta$ and $\mathrm{K}=\mathrm{qvB} / \mathrm{m}$ are constant. In IRE Section $3, \lambda, \mathrm{L} / \mathrm{v}$, and magnet length are held constant, while quadrupole field gradient scales as $1 /(1-2 \eta / 3)^{1 / 2}$ in order to keep $\sigma_{0}$ constant. In this paper we examine the effect of random misalignments of quadrupoles and initial misalignment of the beam on emittance growth. Centroid motion and its dependence on the above scaling are calculated for the present IRE design FODO lattice in Section 2 of the paper. In Section 3 a smooth approximation is used to elucidate the results of Section 2. Section 4 shows the emittance growth due to the misalignments in the presence of image forces and magnet fringe fields, as calculated by PIC simulations.

"Work supported by the U.S. Department of Energy under contracts DE-AC03-76SF00098 (LBNL) and W-7405-ENG-48 (LLNL).

\section{ALTERNATING GRADIENT MODEL}

We consider the motion of the centroid of a transverse slice of the beam, and follow the treatment of L. Smith[1]. A good approximation to the centroid orbit for the present IRE design can be obtained by neglecting image forces and other nonlinearities. Then linearity implies that if the centroid is initially on axis with no transverse velocity, its position at the center of the Nth quadrupole, $x_{N}$, is the sum of the individual perturbations from offsets of the upstream quadrupoles, so that [1]

$$
\begin{aligned}
& x_{N}=2 \sqrt{\frac{\beta_{+N}}{v_{+N}}} \sum_{n=1}^{N}\left\{d_{+n} C_{+n} \sin \left[(N-n) \sigma_{0}\right]\right. \\
&\left.-d_{-n} C_{-n} \sin \left[(N-n) \sigma_{0}-\frac{\sigma_{0}}{2}\right]\right\} .
\end{aligned}
$$

Here subscripts $+n$ and $-n$ refer to evaluation at the nth focusing and defocusing quadrupole, respectively. $d_{+n}$ is the $n$th focusing quad $x$ offset. $C_{+n}=\left(\beta_{+n} K_{+n} / v_{+n}\right)^{1 / 2} \sin \left(\theta_{+n} / 2\right)$, $\theta_{+n}=K_{+n} 1 / 2 \eta L_{+n} / v_{+n}$ and $C_{-n}=\left(\beta_{-n} K_{-n} / v\right)^{1 / 2} \sinh \left(\theta_{-n} / 2\right)$. $\quad \beta$ is the betatron function. We assume that $\mathrm{x}$ and $\mathrm{y}$ are decoupled, so that the same description holds for the $y$ dimension. We are interested in random quadrupole misalignments caused by physical limits on fabrication and alignment, so we assume no correlation between the offsets, and look at statistical behavior of centroid motion averaged over many sets of quad displacements for the lattice (average denoted by "<>"), with the rms "d" for each quad equal to $\mathrm{d}_{\mathrm{rms}}$. Then

$$
\begin{aligned}
<x^{2}>_{N} & =4 \frac{\beta_{+N}}{v_{+N}} d_{r m s}^{2} \sum_{n=1}^{N}<C_{+n}^{2}>\sin ^{2}\left[(N-n) \sigma_{0}\right] \\
& +<C_{-n}^{2}>\sin ^{2}\left[(N-n) \sigma_{0}-\sigma_{0} / 2\right] .
\end{aligned}
$$

In IRE Section 1 , only $\sin ^{2}\left((N-n) \sigma_{0}\right)$ in Eq. (2), and $\left.\sin ^{2}\left[(N-n) \sigma_{0}\right)-\sigma_{0} / 2\right]$, are n-dependent, so the sum is easily done. For $\mathrm{N}\rangle>1$ the dependence of $\mathrm{x}_{\mathrm{N}, \mathrm{rms}}=\left(\left\langle\mathrm{x}^{2}\right\rangle_{\mathrm{N}}\right)^{1 / 2}$ on $\sigma_{0}$ is negligible, and we find that

$$
x_{N, r m s} \approx \zeta_{1} d_{r m s} \sqrt{N},
$$

Thus the centroid motion is to a good approximation (a few percent for $\mathrm{N}>5)$ a random walk. $\zeta_{\mathrm{i}}=\theta_{+\mathrm{i} 0}\left[2 \mathrm{~K}_{+\mathrm{i} 0}\left(\beta_{+\mathrm{i} 0}+\right.\right.$ $\left.\left.\beta_{-i 0}\right)\right]^{1 / 2} / 2$, where the subscript " 0 " refers to an initial value in IRE Section i. For present IRE design parameters, $\zeta_{1}$ is 3.77 , giving $1 \mathrm{~mm}$ centroid offset at the end of the 123 Section 1 lattice periods, for $\mathrm{d}_{\mathrm{rms}}=0.0254 \mathrm{~mm}$ ( $\left.1 \mathrm{mil}\right)$.

In IRE Section 2, the accelerating gradient, $\mathrm{V}_{2}{ }^{\prime}$, increases as $\mathrm{v}^{2}$. We can approximate the gradient as constant over each half lattice period, and equal to the average of the gradient over that length, since $\Delta \mathrm{E} / \mathrm{E} \ll<1$, 
where $\Delta \mathrm{E}$ is the energy increase in a period. Then it is easy to show that $\mathrm{v}$ increases exponentially with $\mathrm{z}$. Using the fact that $\mathrm{L} \propto \mathrm{V} 1 / 2$, one can show that $\mathrm{v}_{\mathrm{n}} \propto\left(1-\mathrm{ng}_{2} \mathrm{~L}_{20}\right)^{-2}$ and $\left(\mathrm{K}_{\mathrm{n}} \beta_{+\mathrm{n}}\right)^{1 / 2} \propto \mathrm{v}{ }^{1 / 2}$, with $\mathrm{g}_{2}=\mathrm{qV}_{2}^{\prime} /\left(\mathrm{mv}^{2}\right)$. This determines the $\mathrm{n}$ dependence of all quantities in Eq. (1). The sum can then be done quite accurately if we expand to 2nd order in $\mathrm{g}_{2}$, since $\mathrm{g}_{2} \approx 0.01$. For $\mathrm{N} \gg>1$, and no original offset or angle in IRE section 2, this gives

$$
x_{N, r m s} \approx \zeta_{2} d_{r m s} \sqrt{N\left(1+\frac{c_{2} N}{2}+\frac{c_{2}^{2} N^{2}}{3}\right)\left(1-c_{2} N\right)} .
$$

$\mathrm{N}$ here is the number of lattice periods traversed in Section 2 , and $\mathrm{c}_{2}=\mathrm{g}_{2} \mathrm{~L}_{20} . \quad \zeta_{2}=3.48$ for the present design. Note that the growth of centroid displacement is considerably slower than $\mathrm{N}^{1 / 2}$. This is due to the decrease of $\beta$, which $\propto \mathrm{v}^{-1 / 2}$. Further insight on this result follows in Section 3.

In similar fashion, using $\mathrm{qV}_{3}^{\prime} /\left(\mathrm{mv}^{2}\right)<<1, \theta_{\mathrm{n}}<<1$, we find for section 3 :

$$
x_{N, r m s} \approx \zeta_{3} d_{r m s} \sqrt{N-1-\frac{1}{3} \eta_{30} Z N^{2}+\frac{2}{9} \eta_{30} Z^{2} N^{3}} .
$$

Here $\mathrm{Z} \equiv \mathrm{g}_{30} \mathrm{~L}_{30} / 2$. For the present design, $\zeta_{3}=3.48$ and $\mathrm{Z}=0.004 \mathrm{~m}$.

The above results have been derived assuming no initial offset or angle, in order to see the scaling for a particular section. They can easily be combined to calculate rms centroid orbit through the accelerator, using the fact that $\mathrm{x}_{\mathrm{N}, \mathrm{rms}}^{\prime} \approx \mathrm{x}_{\mathrm{N}, \mathrm{rms}} / \beta_{+\mathrm{N}}$ for each section, and using the betatron formalism to carry the final offset and angle for IRE Sections 1 and 2 through the rest of the machine. Results agree with computer calculations to about $5 \%$.

\section{SMOOTH APPROXIMATION MODEL}

The calculation of centroid offset in Section 2 is useful for making accurate estimates of centroid evolution. However, it is often useful for accelerator designers to have an even simpler formula that gives more physical insight and is amenable to incorporation into accelerator systems codes. To that end we have used the smooth approximation to represent the centroid orbit, but use the matrix representation of the momentum impulse to calculate the change in amplitude of a particle undergoing kicks from displaced quads. In the absence of quadrupole errors, the xequation of motion in the smooth approximation is:

$$
\mathrm{d}^{2} x / \mathrm{d} t^{2}=-v_{z}^{2} k_{\beta 0^{2}}{ }^{2}
$$

Here $k_{\beta 0} \equiv \sigma_{0} / 2 L$. Letting $j$ denote the number of betatron periods from $z=0$, then $\mathrm{d} j / \mathrm{d} z=k_{\beta 0} / 2 \pi$. Transforming to $j$ as the independent variable, we find

$$
d^{2} x / d j^{2}+\left(d \ln \omega_{\beta O} / d j\right) \mathrm{d} x / \mathrm{d} j+4 \pi^{2} x=0
$$

Here $\omega_{\beta 0} \equiv k_{\beta 0} v_{z}$ is the temporal betatron frequency. From Eq.(7) it is clear that if $\omega_{\beta 0}$ increases with $z$, the amplitude of the betatron motion decreases (in the absence of displacement errors.) We define the amplitude $A$ through the equation $A^{2} \equiv x^{2}+x^{\prime 2} / k_{\beta 0}{ }^{2}$. If follows that

$$
\mathrm{d} A^{2} / \mathrm{d} j+\left(1 / 2 \pi^{2}\right)\left(\mathrm{d} \ln \omega_{\beta O} / \mathrm{d} j\right)(\mathrm{d} x / \mathrm{d} j)^{2}=0
$$

We now consider the change in the amplitude arising from randomly displaced quads. The difference in position $\delta x$ and angle $\delta x^{\prime}$ between the centroid position with and without quad displacement after a focusing quad and drift is

$$
\begin{aligned}
& \delta x=d_{-n}(1-\cos \theta+(1 / \eta-1) \sin \theta) \text { and } \\
& \delta x^{\prime}=d_{-n} \theta \sin \theta /(\eta L)
\end{aligned}
$$

and after a defocusing quad and drift is:

$$
\begin{aligned}
& \delta x=d_{+n}(1-\cosh \theta-(1 / \eta-1) \sinh \theta) \\
& \delta x^{\prime}=-d_{+n} \theta \sinh \theta /(\eta L)
\end{aligned}
$$

The resulting change in amplitude when averaged over different distributions of quad errors is given by $\left\langle\delta A^{2}\right\rangle$ $=\left\langle\delta x^{2}\right\rangle+\left\langle\delta x^{\prime} / k_{\beta 0}{ }^{2}\right\rangle$, where terms linear in $\delta x$ and $\delta x^{\prime}$ average to zero. Averaging Eq.(8) over error distributions and adding effects from Eqs.(9), (10) yields the following evolution equation for the average amplitude squared:

$$
\frac{d}{d i}\left\langle A^{2}\right\rangle=-\left\langle A^{2}\right\rangle \frac{d}{d i} \ln \omega_{\beta 0}+f\left(\sigma_{0}, \eta\right)\left\langle d_{ \pm}^{2}\right\rangle
$$

Here $i$ is a half lattice period (one quad and drift) and $\mathrm{f}\left(\sigma_{0}, \eta\right) \equiv(1 / 2)\left[(1-\cos \theta+(1 / \eta-1) \sin \theta)^{2}+(1-\cosh \theta-((1 / \eta-\right.$ 1) $\left.\sinh \theta)^{2}+\left(2 \theta / \eta \sigma_{0}\right)^{2}\left(\sin ^{2} \theta+\sinh ^{2} \theta\right)\right] . \quad \theta \approx\left[6 \eta^{2}\left(1-\cos \sigma_{0}\right) /(3-\right.$ $2 \eta)]^{1 / 4}$ (ref. [2]). Since $f\left(\sigma_{0}=0, \eta\right)=12 /(3-2 \eta)$ and variation due to $\sigma_{0}$ is small relative to that from $\eta$, for some purposes the $\sigma_{0}=0$ value of $f\left(\sigma_{0}, \eta\right)$ is adequate.

In order to compare the results from integrating Eq. (11) to the results obtained from transfer matrices, we need to evaluate the "smooth" component from each AG orbit. This is accomplished by averaging over the lattice period using the following procedure. We assume that the actual position after $\mathrm{i}$ half-lattice periods may be expressed as $x(i)=A \cos \left(\sigma_{0} i / 2+\phi\right)+x_{\mathrm{L}} \cos (\pi i)$. We define the quantities $\bar{x}(i) \equiv(1 / 4)[x(i-1)+2 x(i)+x(i+1)] \quad$ and $\bar{x}^{\prime}(i) \equiv(1 / 4)\left[x^{\prime}(i-1)+2 x^{\prime}(i)+x^{\prime}(i+1)\right]$. The smooth amplitude is then given by

$$
A^{2}(i)=4\left(\bar{x}(i)^{2}+\bar{x}^{\prime}(i)^{2} / k_{\beta 0}^{2}\right) /\left(1+\cos \left(\sigma_{0} / 2\right)\right)^{2}
$$

We compare integration of Eq. (11) with the numerical amplitude found using Eq. (12) in figure (1).

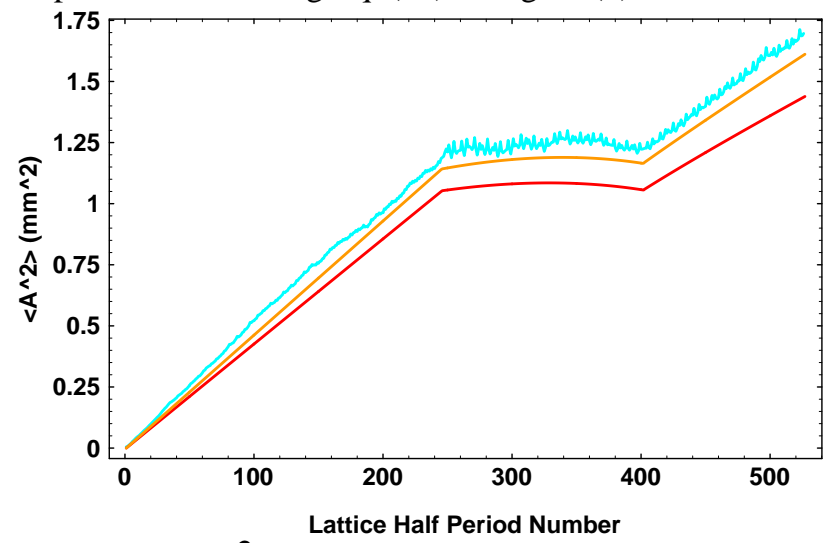

Figure (1): $\left\langle A^{2}\right\rangle$ vs. half-lattice number $i$, calculated using Eq.(12) (upper), Eq. (11) (middle), and using the $\sigma_{0}=0$ value of $f\left(\sigma_{0}, \eta\right)$ (lower). 
From Eq. (11) it is apparent that increasing $\omega_{\beta 0}$ with $z$ helps to damp out betatron oscillations and the question arises as to whether advantage can be taken of this in accelerator design. We note that consideration of the smooth envelope equation places the constraint that $\omega_{\beta 0}$ is proportional to $\lambda / a^{2}$ and for designs in which the beam radius $a$ is constant $\omega_{\beta 0}$ varies directly with the line charge $\lambda$, suggesting that damping will generally accompany bunch compression. Apertures and bunching schedules should be chosen bearing in mind this relation.

\section{PIC SIMULATION RESULTS FOR OFF-AXIS BEAMS}

Simulations using the $2 d$ (transverse) version of the WARP particle-in-cell code were done to investigate the behavior of initially aligned and misaligned beams in the IRE for the case of random quad misalignments. In order to incorporate the effects of image charges on the beam, the presence of conducting boundaries in the three sections of the accelerator was included in the simulation. The aperture of the accelerator was $2.86 \mathrm{~cm}$ in IRE Section 1, and was increased to $3.36 \mathrm{~cm}$ in IRE Sections 2 and 3 . In IRE Section 1, the focusing field is generated by four perfectly conducting electrostatic quadrupole rods with a radius of $3.27 \mathrm{~cm}$. The distance from the axis of the accelerator to the tip of the quadrupoles was equal to the aperture of $2.86 \mathrm{~cm}$. The boundary in the drift spaces between the quadrupoles was a square conducting box, $12.26 \mathrm{~cm}$ on a side. In Sections 2 and 3 , instead of four quadrupole rods, a circular conducting boundary of $3.36 \mathrm{~cm}$ was used both in the quadrupoles and in the drift spaces.

A beam of $\mathrm{K}^{+}$ions was accelerated from $1.6 \mathrm{MeV}$ to $200 \mathrm{MeV}$. $\sigma_{0}$ was set equal to $70^{\circ}$, average radius was 1.5 $\mathrm{cm}$, and the perveance was $1.4 \times 10^{-3}$.

The rms random quadrupole offset was $0.0254 \mathrm{~mm}$ for each of the transverse directions. In the simulations in which the beam was initially misaligned, it was initially off axis by $0.8 \mathrm{~mm}$ in one of the transverse directions at the beginning of the first drift space. This resulted in an initial beam centroid oscillation of $2 \mathrm{~mm}$ in amplitude.

In real $\mathrm{AG}$ focusing systems, since the quadrupole field strength changes near the magnet ends, pseudo-octupole and pseudo-dodecapole fringe fields there will act nonlinearly on the beam [3]. These fringe fields were included in a subsequent simulation, assuming that the quadrupole field strength decreases smoothly near the magnet edge with a fall-off length of $4.5 \mathrm{~cm}$. This length fills most of the drift space in the first few lattice periods, but avoids overlap of the fields from one quad to the next.

If the fringe fields are included in the simulation, the emittance growth is still small. Since the stepsize has to be much smaller in order to resolve the fringe fields, the simulation needed a much longer running time $(67,500$ user CPU seconds on a Cray J90).

The results of simulations using 80,000 particles on a 256 × 256 grid are shown in Table 1 and Figure 2. The emittance behavior was alike in both transverse directions, due to coupling by nonlinearities. Maximum centroid offset was approximately $1.5 \mathrm{~mm}$, which is consistent with the results of Sections 2 and 3 of this paper.

Table 1: PIC simulation results

\begin{tabular}{|c|c|c|}
\hline Case & $\begin{array}{c}\text { Particles } \\
\text { los t }\end{array}$ & $\begin{array}{c}\text { Emittance } \\
\text { growth }\end{array}$ \\
\hline $\begin{array}{c}\text { Misaligned quads, } \\
\text { no fringe fields (a) }\end{array}$ & 3 & $4.1 \%$ \\
\hline $\begin{array}{c}\text { Misaligned beam, } \\
\text { no fringe fields (b) }\end{array}$ & 5 & $4.8 \%$ \\
\hline $\begin{array}{c}\text { Misaligned beam, } \\
\text { with fringe fields (c) }\end{array}$ & 3 & $5.2 \%$ \\
\hline $\begin{array}{c}\text { Misaligned quads, } \\
\text { misaligned beam, } \\
\text { no fringe fields (d) }\end{array}$ & 42 & $5.9 \%$ \\
\hline
\end{tabular}



Figure 2: Increase in the normalized transverse emittance in case of misalignments in the IRE.

We conclude that moderate misalignments in the IRE do not lead to any significant emittance growth. These are encouraging results for beam stability in the IRE.

\section{CONCLUSIONS}

We have derived very accurate formulas for the statistical behavior of the beam centroid, given various practical scalings of lattice parameters, and shown that increasing $\lambda / a^{2}$ with $\mathrm{z}$ can produce damping of the centroid offset. These results can be used as design guidance, and to estimate necessary aperture, given fabrication and alignment tolerances. PIC simulations for a preliminary IRE design show very small emittance growth $(\sim 5 \%)$ due to beam and quadrupole misalignments for $\mathrm{rms}$ quad displacement of $0.0254 \mathrm{~mm}$.

\section{REFERENCES}

[1] L. Smith, "Effect of Random Quadrupole Misalignments in MBE", HI-FAN-251, July 1984, unpublished.

[2] E.P. Lee, T.J. Fessenden, and L. J. Laslett, "Transportable Charge in a Periodic Alternating Gradient System,"IEEE Trans Nucl. Science, NS-26, 2489 (1989).

[3] E. P. Lee, "Field Calculation for a $\cos (2 \theta)$ Quadrupole Magnet Without Iron", HI-FAR-464, July 1996, unpublished. 